\section{Hemoglobin Kinetics and the Effect of Organic Phosphates}

Abstract. A kinetic model based on allosteric mechanisms of cooperativity fits the experimentally observed phosphate dependence of hemoglobin reactions. Subunit inequivalence is found to be important in analyzing hemoglobin kinetics. The observed increase in the rate of deoxygenation in the presence of organic phosphates is primarily related to the increased rate of dissociation of the second oxygen molecule.

The kinetics of the reactions of hemoglobin $(\mathrm{Hb})$ with ligands are generally interpreted by using an Adair scheme, with eight rate constants (1); this scheme applies to situations with rapid conformational equilibria and identical subunits. The Adair scheme, when extended to allow for inequivalent subunits (2), has 32 rate constants. Other kinetic models $(3,4)$ which are based on allosteric mechanisms for cooperativity do not allow for inequivalent subunits. All previous work has required a different set of rate constants for each set of experimental conditions (such as $p \mathrm{H}$ or phosphate concentrations).

The model we have developed (5) [based on a recently proposed equilibrium model (6)] allows for inequivalent subunits and combines different allosteric mechanisms for homotropic and heterotropic cooperativity. It is applied here to study the effect of 2,3-diphosphoglycerate (DPG), inositol hexaphosphate (IHP), and 8-hydroxy-1,3,6-pyrenetrisulfonate (HPT) on the kinetics of $\mathrm{Hb}$ deoxygenation and oxygenation. For a given phosphate a single set of parameters is sufficient to describe kinetic data over a wide range of phosphate concentrations.

The mechanism for homotropic cooperativity being considered corresponds to that of Perutz (7) (induced-fit binding of $\mathrm{O}_{2}$ and conformational constraints), although we need not assume a fixed sequence of oxygenation, or a fixed point at which the quaternary conformation switches from the deoxy to the oxy form. The binding of organic phosphates is considered to shift the quaternary equilibrium and to alter the strength of the conformational (quaternary) constraints (6).

We assume that (i) the quaternary equilibrium and (ii) the equilibria of DPG, IHP, and HPT binding to $\mathrm{Hb}$ are rapid compared to the $\mathrm{O}_{2}$ binding process. Evidence in support of (i) comes from flash photolysis experiments (8), while (ii) is consistent with the fact that preincubation of $\mathrm{Hb}$ with DPG (9) [or IHP (10) or HPT (11)] leads to the same kinetics as observed when the two are mixed in a stopped-flow apparatus.

Figure $1 \mathrm{~A}$ shows the equilibrium between the deoxy and oxy quaternary conformations of unliganded $\mathrm{Hb}$, with equilibrium constant $K_{Q}$. Equilibria similar to that shown in Fig. 1A exist at every stage of ligand binding. The conformational equilibrium constant in the general case becomes

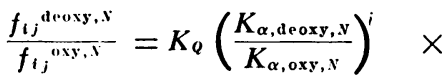

$$
\begin{aligned}
& \left(\frac{K_{\beta, \text { leoxy }, N}}{K_{\beta, 0 x y, N}}\right)^{j} \quad\left(\frac{\tilde{K}_{\mathrm{deoxy}}}{\tilde{K}_{\text {oxy }}}\right)^{x}
\end{aligned}
$$

Here $f_{i j}^{Q N}$ denotes the fraction of molecules that are in the $Q$ quaternary conformation ( $Q=$ deoxy or oxy) and have $N$ phosphate molecules bound $(N=0$ or 1$), i$ liganded $\alpha$ subunits, and $j$ liganded $\beta$ subunits $(i, j=0,1,2$ for $\mathrm{Hb})$; and $K_{a, Q N}$ denotes the equilibrium constant for ligand binding to an $\alpha$ subunit of a molecule in the $Q$ quaternary conequilibrium constants defined above. formation with $N$ bound phosphate molecules. As shown in Fig. $1 \mathrm{~B}, \tilde{K}_{\text {deoxs }}$ and $\tilde{K}_{\text {oxy }}$ are the equilibrium constants for binding of organic phosphate, $\mathbf{P}$, to unliganded $\mathrm{Hb}$ in the deoxy and oxy quaternary conformations, respectively. Equilibria similar to those shown in Fig. $1 B$ exist at every stage of ligand binding, and the equilibria are described by equations analogous to Eq. 1A

$$
\frac{f_{i j}^{Q 1}}{f_{i j}^{Q 0}}=[\mathrm{P}]_{\mathrm{rec}} \tilde{K}_{Q}\left(\frac{K_{\alpha, Q 1}}{K_{\alpha, Q 0}}\right)^{i} \quad\left(\frac{K_{\beta, Q 1}}{K_{\beta, Q 0}}\right)^{i}
$$

where the square brackets indicate concentration.

Figure $1 \mathrm{C}$ defines the off and on rate constants, $k_{\alpha, Q N}$ and $k_{\alpha, Q N}^{\prime}$, for ligand binding to an $\alpha$ subunit of the Hb tetramer in the $Q$ quaternary conformation with $N$ phosphate molecules bound. The kinetics are obtained by using the "master equation" approach $(4,12)$, which allows us to decompose the off and on rates into equilibrium and kinetic factors

$$
\begin{array}{r}
k_{\alpha, Q N}^{\prime}=\left[1-\phi(Q) \theta_{\alpha}(N)\right] \omega_{\alpha} \\
k_{\alpha, Q x}^{\prime}=\left[1+\phi(Q) \theta_{\alpha}(N)\right] \omega_{\alpha} K_{\alpha}
\end{array}
$$

(The rate constants for the $\beta$ subunits can be similarly decomposed.) Here $\phi(Q)= \pm 1$ for the oxy and deoxy quaternary conformations, respectively;

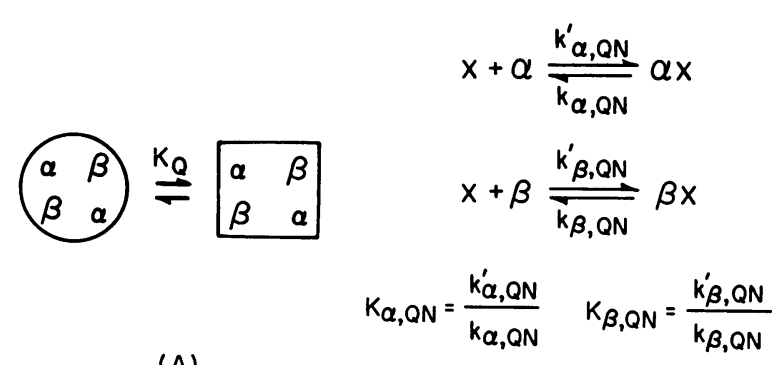

(A)

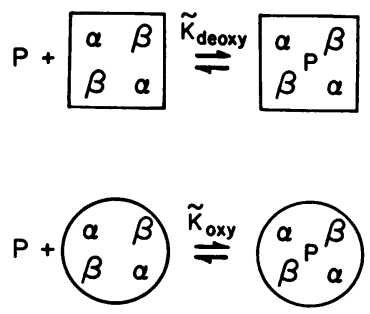

(B)

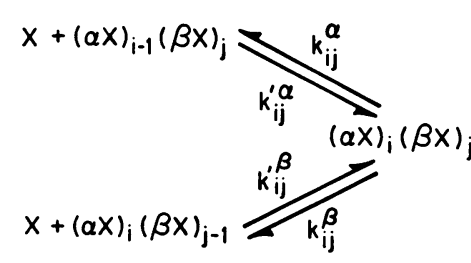

(D)

Fig. 1. The special case of the model which we apply here to $\mathrm{Hb}$ includes rapid quaternary conformation changes ( $A$, short arrows), rapid phosphate binding (B, short arrows), and slow oxygen binding ( $\mathrm{C}$, long arrows). (A) Equilibrium between deoxy and oxy quaternary conformations of unliganded $\mathrm{Hb}$, with equilibrium constant $K_{\mathbf{Q}}$. (B) Equilibria for phosphate $(P)$ binding to unliganded $\mathrm{Hb}$ in the deoxy and oxy quaternary conformation, with equilibrium constants $\tilde{K}_{\text {deoxy }}$ and $\tilde{K}_{\text {oxy }}$, respectively. (C) Reactions for ligand (X) binding to individual $\alpha$ and $\beta$ subunits in a molecule which is in the $Q$ (deoxy or oxy) quaternary conformation and has $N$ ( 0 or 1$)$ phosphate molecules bound. For the $\alpha$ subunits, the on and off rate constants are denoted by $k_{\alpha, Q N}^{\prime}$ and $k_{\alpha, Q N}$, respectively, and the equilibrium constant by $K_{\alpha . Q v}$. Similar notation is used for the $\beta$ subunit. For a fixed phosphate concentration this model is equivalent to the extended Adair model (2) shown in (D). The overall rates $k_{i j}^{\alpha}, k_{i j}^{\prime}, k_{i j}^{\beta}$, and $k_{i j}^{\beta}$ are functions of the rate constants and 
$\theta_{\alpha}(N)$ is related to the strength of the quaternary constraints through $\theta_{\alpha}(N) \equiv$ $\tanh \left[U_{Q T}^{\alpha}(N) / k_{\mathrm{B}} T\right]$, where $k_{\mathrm{B}} T$ is the product of the Boltzmann constant and the temperature, and $U_{Q T}^{\alpha}(N)$ is the energy of the conformational constraints on an $\alpha$ subunit in the deoxy tertiary conformation when the molecule is in the oxy quaternary conformation (6). Therefore, $K_{\alpha, Q N} \equiv k_{\alpha, Q N}^{\prime} / k_{\alpha, Q N}=$ $K_{\alpha} \exp \left[2 \phi(Q) U_{Q T}^{\alpha}(N) / k_{\mathrm{B}} T\right], \quad$ consistent with equations 34 and 37 of (6). Note that in the absence of quaternary constraints, $\theta_{\alpha}(N)=0$ and $K_{\alpha}$ is the equi- librium constant for induced-fit binding of the ligand to a single $\alpha$ subunit. If $\theta_{\alpha}(1)=\theta_{\alpha}(0)$, then the organic phosphate does not alter the strength of the quaternary constraints and its inhibitory effect is solely due to its preferential binding to the two quaternary conformations. The parameter $\omega_{\alpha}$ has no equilibrium analog; it represents the off rate from an $\alpha$ subunit in the absence of quaternary constraints. In principle, $\omega_{\alpha}$ could be dependent on the quaternary state and the presence of effectors. These possibilities have been systematically explored in fitting the theory to experimental data.

If the $\alpha$ and $\beta$ subunits are considered kinetically equivalent, then $k^{\prime}{ }_{0 x y, N=0}$ and $k_{0 x y, N=0}$ can be estimated by measuring the rate constants for the binding of the fourth ligand molecule in the absence of phosphates. Similarly, $k_{\text {deoxy, } N}^{\prime}=0$ and $k_{\text {deoxy, } N}=0$ can be obtained from the rate constants for the binding of the first ligand molecule (3). Estimates of $k_{Q, N}^{\prime}=1$ can be similarly obtained from analyzing the reactions at very high organic
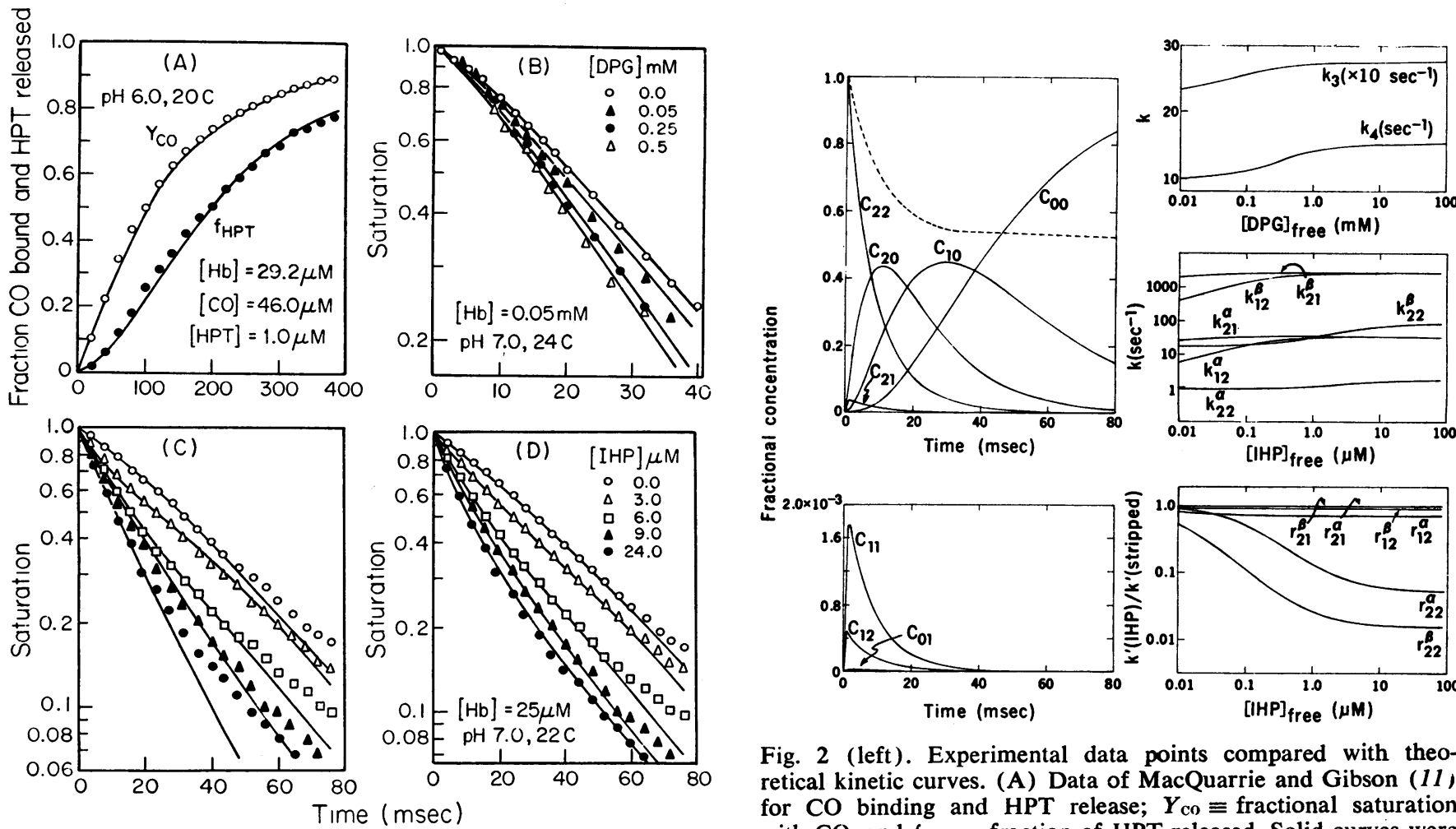

Fig. 2 (left). Experimental data points compared with theoretical kinetic curves. (A) Data of MacQuarrie and Gibson (11) for $\mathrm{CO}$ binding and HPT release; $\boldsymbol{Y}_{\mathrm{co}} \equiv$ fractional saturation with $\mathrm{CO}$, and $f_{11}{ }^{\prime} \mathrm{T} \equiv$ fraction of $\mathrm{HPT}$ released. Solid curves were calculated assuming identical subunits $(\alpha=\beta)$, with $\omega_{\alpha} K_{\alpha}=9.8$ liter $\mu \mathrm{mole}^{-1} \mathrm{sec}^{-1}, \theta_{\alpha}=0.9848, K_{Q}=2.71 \times 10^{12}, \widetilde{K}_{\text {oxy }}=5.26 \times 10^{\prime}$ liter $/$ mole, and $\tilde{K}_{\text {deoxy }}=9.1 \times 10^{4}$ liter/mole. (B) Data of Salhany et al. (13) for deoxygenation of oxy-Hb by dithionite in the presence of DPG. Curves were calculated assuming identical subunits, with $\omega_{\alpha}=147.5 \mathrm{sec}^{-1}, \theta_{\alpha}=0.9403, K_{Q}=4.468 \times 10^{\prime \prime}, \tilde{K}_{\text {oxy }}=3.2$ $\times 10^{3}$ liter/mole, and $\widetilde{K}_{\text {deoxy }}=2.13 \times 10^{4}$ liter/mole. (C) Data of Gray and Gibson (10) for deoxygenation of oxy-Hb by dithionite $\left(\mathrm{Na}_{2} \mathrm{~S}_{2} \mathrm{O}_{4}\right)$ in the presence of IHP. Theoretical curves were calculated with $\omega_{\alpha}=556 \mathrm{sec}^{-1}, \theta_{\alpha}=0.9866, K_{Q}=3.08 \times 10^{11}$ $\tilde{K}_{\text {oxy }}=0.94 \times 10^{5}$ liter $/$ mole, and $\widetilde{K}_{\text {deoxy }}=7.26 \times 10^{s}$ liter $/$ mole. (D) Reaction of Fig. $2 \mathrm{C}$ calculated by assuming inequivalent subunits $(\alpha \neq \beta)$. The theoretical parameters are $\omega_{\alpha}=18 \mathrm{sec}^{-1}, \omega_{\beta}=1384 \mathrm{sec}^{-1}, \theta_{\alpha}(0)=\theta_{\alpha}(1)=0.9496, \theta_{\beta}(0)=\theta_{\beta}(1)=0.9873, K_{2}=$ $5.327 \times 10^{10}, \tilde{K}_{\text {oxy }}=2.69 \times 10^{5} \mathrm{liter} / \mathrm{mole}$, and $\tilde{K}_{\mathrm{deoxy}}=1.83 \times 10^{8} \mathrm{liter} / \mathrm{mole}$. In the data shown in (C) and (D) some of the Hb may be present as dimers ( $<10$ percent, using $[\mathrm{Hb}]=50 \mu M$ before mixing). With increasing IHP, the fraction of dimers will decrease. The disagreement between calculation and experiment near the end of the reaction could be partly due to slow absorbance changes involving dimers. Fig. 3 (right). Time dependence of the concentrations of the various species produced in the reaction of Fig. 2, C and D, and the effect of organic phosphates on the rate constants. (A) Fractional concentrations of various liganded species, $C_{i j}=\left[(\alpha x)_{i}(\beta x)_{j}\right] /\left[\left[_{b}\right]_{t}\right]_{t a t a l}$ and $[I H P]_{\text {trec }} /[I H P]_{\text {trew at } t=0}$ (dashed line) as a function of time, calculated by using the parameter values of Fig. 2D. Total IHP $=9.0 \mu M$ and total $\mathrm{Hb}_{4}=6.25 \mu M$ (by tetramer). The value of $C_{0: 2}$ is less than $10^{-5}$ and cannot be displayed on either scale. (B, top) Dependence of $k_{4}$ and $k_{3}$ on [DPG] trie, calculated by using the parameters of Fig. 2B. The rates $k_{1}=k_{2}=286 \mathrm{sec}^{-1}$ were not significantly increased (see text). (B, middle) Effect of [IHP] $]_{\mathrm{rren}}$ on the off rates $k_{2: 2}, k_{21}$, and $k_{1:}$ for the $\alpha$ and $\beta$ subunits, calculated from the parameters of Fig. 2D. The subunit with the greater value of $k_{*: 2}$ is identified as the $\beta$ subunit, in accordance with previous findings (18). Here again, the off rates from species with at most two hemes liganded are not significantly altered by IHP; $k_{10}^{\alpha}=k_{v 1}^{\alpha}=k_{11}^{\alpha}=35.6 \mathrm{sec}^{-1} ; k_{01}^{\beta}=k_{(v 2}^{\beta}=k_{11}^{\beta}=2750 \mathrm{sec}^{-1}$. (B, bottom) Calculated variation in the ratio $r_{i j}^{\alpha} \equiv k_{i j}^{\prime}(\mathrm{IHP}) / k_{i j}^{\prime \alpha}(\mathrm{IHP}=0)$, and $r_{i j}^{\beta} \equiv k_{i j}^{\alpha}(\mathrm{IHP}) / k_{i j}^{\prime \beta}(\mathrm{IHP}=0)$ with [IHP] $\mathrm{frer}_{\text {, for }} i+j \geq 3$. This prediction should be amenable to experimental verification. The concentration of DPG is in millimoles per liter and that of IHP in micromoles per liter. All rate constants are expressed on a per heme basis. We have calculated the relative probabilities of the deoxy and oxy quaternary conformations in the Hb molecule with $n$ hemes deoxygenated. Using the theoretical parameters given in Fig. 2B for DPG and con Fig. 2D for IHP, we find that the switch from the oxy to the deoxy quaternary conformation occurs just after or just before the deoxygenation of the second heme, depending on the phosphate concentration. Thus the change in $k_{i n}, k_{12}$, and $k^{\prime}$ both $\alpha$ and $\beta$ subunits) with increasing phosphate concentration is related to the fact that the presence of phosphates has its strongest effect on the quaternary conformation of $\mathrm{Hb}$ with three hemes liganded. 
phosphate concentrations. These estimates of $k_{Q, N}^{\prime}$ and $k_{Q, N}$ can, in principle, be used to determine the parameters $\omega, K$, and $\theta(N)$. For the case of inequivalent subunits, estimates of $k_{\alpha, Q N}^{\prime}$ and $k_{\alpha, Q N}$ would be required to determine $\omega_{\alpha}, K_{\alpha}$, and $\theta_{\alpha}(N)$. The organic phosphate binding constants $\tilde{K}_{\text {oxy }}$ and $\tilde{K}_{\text {deoxy }}$ can be obtained by direct measurement $(10,11)$. The remaining parameter, $K_{Q}$, can be obtained from fitting equilibrium oxygenation data (3). For the kinetic data analyzed here, we have determined all the parameters by a least squares fitting procedure.

Figure $2 \mathrm{~A}$ shows data (11) for the binding of $\mathrm{CO}$ and consequent release of HPT, a fluorescent analog of DPG. By treating the subunits as equivalent and neglecting the $\mathrm{CO}$ off rates, we can fit both sets of data to better than 1 percent per point by using a single set of five parameters $\left(\omega_{\alpha} K_{\alpha}\right.$ $\theta_{\alpha}, K_{Q}, \tilde{K}_{\text {orr }}$ and $\left.\tilde{K}_{\text {deoxy }}\right)$; here $\theta_{\alpha} \stackrel{\alpha}{\equiv}$ $\theta_{\alpha}^{\alpha}(1)=\theta_{\alpha}(0)$. MacQuarrie and Gibson (11) require nine parameters to explain these data-four $\mathrm{CO}$ rate constants and five HPT equilibrium constants.

Figure 2B shows data (13) for the release of $\mathrm{O}_{2}$ from oxy-Hb in the presence of varying DPG concentrations. The $\left[\mathrm{O}_{2}\right]_{\text {free }}$ at $t=0$ and $\left[\mathrm{Na}_{2} \mathrm{~S}_{2} \mathrm{O}_{4}\right]$ were such that $\left[\mathrm{O}_{2}\right]_{\text {free }}$ becomes negligible in $<5 \mathrm{msec}$ (14). Thus, only the off rates contribute to the reaction. The five parameters $\left(\omega_{\alpha}, \theta_{\alpha}, K_{Q}, \tilde{K}_{\text {oxy }}\right.$, and $\tilde{K}_{\text {deoxy }}$ ) were found from the fit to the data for 0 and $0.5 \mathrm{mM}$ DPG, and these same parameters were then used to calculate the curves for 0.05 and $0.25 \mathrm{mM}$ DPG. The agreement of these calculated curves with the data gives us confidence in the predictive ability of our approach. No further improvements in fit were obtained by considering the possibilities of (i) inequivalent subunits $(\alpha \neq \beta)$, (ii) DPG having an effect on the quaternary constraints, and (iii) the time scaling parameter, $\omega$, being dependent on the quaternary conformation or DPG binding. Note that the Adair scheme (1) requires four off rate constants for each DPG concentration, making a total of 16 parameters for the data of Fig. 2B. If the equilibrium scheme of Tyuma et al. (15) is extended to kinetics, a total of 13 parameters is required for the deoxygenation data, and a total of 21 parameters for oxygenation data.

Figure 2, C and D, show deoxygenation data $(10)$ in the presence of IHP.
A slight improvement over the fiveparameter fit (Fig. 2C) was obtained by considering IHP to alter the quaternary constraints, whereas the effects of considering $\omega_{\alpha}$ and $\omega_{\beta}$ to depend on quaternary conformation and IHP binding were insignificant. A somewhat improved fit (Fig. 2D) was obtained by allowing for $\alpha \neq \beta$ (seven parameters). These results suggest that the $\alpha$ and $\beta$ subunits may be kinetically inequivalent in the presence of IHP. That this inequivalence does not appear with DPG may be due to the fact that DPG does not affect the limiting rate constant $k_{4}=\left(k_{22}^{\alpha}+k_{22}^{\beta}\right) / 2$, whereas IHP does.

Figure 3A shows the time dependence of $[I H P]_{\text {free }}$ and the concentrations of the variously liganded species produced in the course of the reaction. Note that the concentrations of the intermediate species are very small when compared with the fully liganded and fully unliganded species. A comparison of the fraction of $\mathrm{O}_{2}$ released with the fraction of IHP bound, at every instant of time, shows that the release of $\mathrm{O}_{2}$ lags behind the binding of IHP. This lag is related to the switchover to the deoxy conformation when only three hemes are liganded. (The same effect is less pronounced for DPG.) This result agrees with the similar prediction for DPG based on ${ }^{31} \mathrm{P}$ nuclear magnetic resonance spectra (16), as well as with the results shown in Fig. 2A (11).

The kinetic equations for the model shown in Fig. 1 reduce to the Adair form $(1,2)$ when the conformational equilibria and the organic phosphate binding equilibria are rapid. If $\alpha \neq \beta$, the 32 rate constants of the OlsonGibson reaction scheme (2) are given by

$$
\begin{aligned}
k_{i j}^{\alpha} & =\sum_{Q} \sum_{N=0}^{1} f_{i j}^{Q N} k_{\alpha, Q,} \\
k^{\prime \alpha} & =\sum_{Q} \sum_{N=0}^{1} f_{i-1, j}^{Q N}, k_{\alpha, Q N}^{\prime}
\end{aligned}
$$

where $i=1,2$ and $j=0,1,2$. The rates $k_{i j}^{\beta}$ and $k_{i j}^{\prime \beta}$ can be expressed similarly. The $f_{i j}^{Q N}$ can be obtained from Eqs. 1A and $1 \mathrm{~B}$ by applying the constraint $\Sigma_{Q} \Sigma_{N} f_{i j}^{Q N}=1$. [All rate constants are expressed on a per heme rather than a per tetramer basis (2).] If $\alpha=\beta$, the eight off and on rates, $k_{n}$ and $k_{n}^{\prime}(n=1$, $\ldots, 4)$, for the $\alpha=\beta$ Adair scheme (1) are obtained from Eq. 2 by setting $k_{n=i+j}=k_{i j}^{\alpha}, \quad k_{n=i+j}^{\prime}=k_{i j}^{\prime \alpha}$, and $f_{n}^{Q N}=\Sigma_{i, j}^{i+j}=n f_{i j}^{Q N}$. Thus at a fixed phosphate concentration these eight rate constants can be expressed as functions of the four parameters $\omega, \theta(0), K$, and $K_{Q}$. If $\alpha \neq \beta$, the 32 rate constants can be expressed as functions of the seven parameters $\omega_{\alpha}, \omega_{\beta}, \theta_{\alpha}(0), \theta_{\beta}(0), K_{\alpha}, K_{\beta}$, and $K_{Q}$. In addition, for a rapidly equilibrating phosphate, the parameters $\widetilde{K}_{\text {oxy }}$, $\tilde{K}_{\text {deoxy }}, \theta_{\alpha}(1)$, and $\theta_{\beta}(1)$ are sufficient to describe the phosphate dependence of the rate constants.

Experiments show that the overall dissociation velocity increases in the presence of organic phosphates. Since the reaction consists of many steps it is not possible to tell experimentally which step is most affected by phosphates. However, a theoretical analysis of the data can be used to predict which step is affected most. For this purpose the phosphate dependence of the Adair rate constants is calculated and the results are shown in Fig. 3B. Whereas DPG causes only a small increase in $k_{4}$, IHP has a significant effect on $k_{2:}^{\beta}$. The largest effect of phosphates is on the off rate for the release of the second $\mathrm{O}_{2}$ molecule from fully liganded $\mathrm{Hb}$ (that is, $k_{3}$ or $k_{21}$ and $k_{12}$ ). In the case of IHP, with inequivalent subunits $(\alpha \neq \beta)$, the increase is greatest for dissociation from the species with one $\alpha$ and two $\beta$ subunits liganded. It is the dependence of $k_{22}, k_{21}$, and $k_{12}$ on $[\mathrm{IHP}]_{\text {free }}$ which causes biphasic deoxygenation when $[\mathrm{IHP}]_{\text {free }}$ decreases significantly during the reaction.

If the phosphates do not alter the quaternary constraints and the kinetic parameter $\omega$, then $k_{10}^{\alpha}$ and $k_{01}^{\beta}$ would not be significantly altered by these effectors. Hence dependence of these rate constants (and the equilibrium constants $K_{10}^{\alpha}$ and $K_{01}^{\beta}$ ) on phosphate concentration would indicate an effect of phosphates on the strength of the quaternary constraints and the parameter $\omega$. The calculations show that the $k_{i j}$ with $i+j \leq 2$ are at least an order of magnitude larger than $k_{22}^{\alpha}$ and $k_{22}^{\beta}$. Since in the experiments analyzed here only very small quantities of partially liganded species are produced, $k_{i j}(i+j \leqslant 2)$ cannot be determined very accurately.

The ratio of the on rates $k_{i j}^{\prime}$ (phosphates) $/ k_{i j}^{\prime}$ (stripped) can be calculated by using the parameters determined here. This ratio decreases as phosphate concentration increases, in agreement with the results of Gibson (17). As can be seen from Fig. 3B, $k_{22}^{\prime^{\beta}}$ decreases most, and $k_{22}^{\prime \alpha}$ and $k_{12}^{\prime \alpha}$ decrease somewhat less. The decrease of $k_{22}^{\prime \alpha}$ and $k_{22}^{\prime \beta}$ in the presence of IHP agrees with the measured decrease in 
$k_{4}^{\prime}(10)$. In contrast to the result of Gibson (17), we find that $k^{\prime}{ }_{4}$ decreases with increasing DPG.

The results of our calculation show that (i) the major kinetic effects of different quaternary conformations and of phosphate binding are reproduced sufficiently well by considering simply quaternary constraints and preferential binding; (ii) the inequivalence of subunits $(\alpha \neq \beta)$ is important in analyzing deoxygenation data in the presence of IHP; (iii) the observed increase in the "overall" deoxygenation rate in the presence of IHP largely results from the increase in the rate of dissociation of the second molecule, and this is related to the switchover to the deoxy quaternary conformation at this stage of the reaction; (iv) the calculated values of $\tilde{k}_{4}, \tilde{k}_{2,}^{\beta}$, and the binding constants $K_{\text {oxy }}$ and $K_{\text {deoxy }}$ for HPT, DPG, and IHP are in agreement with previously determined values $(10,11,17,18)$; and (v) our value of $k_{22}^{\alpha}$ is somewhat lower than that determined by Olson et al. (18). While we believe that $k_{3}$ is weli determined in this analysis, the values of $k_{1}$ and $k_{2}$ can only be considered to be estimates. Our results point to the need for an accurate set of data at various DPG (or IHP) levels from $\mathrm{O}_{2}$ binding experiments and from experiments where significant quantities of intermediates are produced (19). The successful use of the master equation formulation to predict the kinetics of hemoglobin reactions suggests the possible use of this approach to the kinetics of other cooperative proteins.

Rama Bansil*, Judith Herzfeld $\dagger$ H. Eugene Stanley

Harvard-MIT Program in

Health Sciences and Technology and

Department of Physics,

Massachusetts Institute of

Technology, Cambridge 02139

\section{References and Notes}

1. Q. H. Gibson and F. J. W. Roughton, Proc. R. Soc. Lond. Ser. B Biol. Sci. 146, 206 (1957).

2. J. S. Olson and Q. H. Gibson, J. Biol. Chem. 247, 1713 (1972).

3. J. J. Hopfield, R. G. Shulman, S. Ogawa, J. Mol. Biol. 61, 425 (1971).

4. R. T. Bush and C. J. Thompson, Biopolymers 10, 961 and 1331 (1971).

5. R. Bansil, thesis, University of Rochester (1974).

6. J. Herzfeld and H. E. Stanley, J. Mol. Biol. 82, 231 (1974); Biochem. Biophys. Res. Commun. 48, 307 (1972).

7. M. F. Perutz, Nature (Lond.) 228, 726 (1970).

8. B. Alpert, R. Banerjee, L. Lindqvist, Proc. Natl. Acad. Sci. U.S.A. 71, 558 (1974); J. A. McCray, Biochem. Biophys. Res. Commun. 47, 187 (1972).

9. Q. H. Gibson, Biochem. Biophys. Res. Commun. 40, 1319 (1970)
10. R. D. Gray and Q. H. Gibson, J. Biol. Chem. 246, 7168 (1971).

11. R. MacQuarrie and Q. H. Gibson, ibid. 247, 5686 (1972).

12. R. J. Glauber, J. Math. Phys. 4, 294 (1963). For applications of this approash to cooperative rhenomena in other systems, see, for example, H. E. Stanley, Introduction to Phase Transitions and Critical Phenomena (Oxford Univ. Press, London, 1971).

13. J. M. Salhany, R. S. Eliot, H. Mizukami, Biochem. Biophys. Res. Commun. 39, 1052 (1970).

14. The upper limit of $5 \mathrm{msec}$ is arrived at by assuming that the reaction of $O_{\text {, with }}$ $\mathrm{Na}_{2} \mathrm{~S}_{2} \mathrm{O}_{4}$ is zero order in $\mathrm{O}_{2}$ and first order in $\mathrm{Na}_{2} \mathrm{~S}_{2} \mathrm{O}_{4}$ [J. A. Morello, M. R. Craw, H. P. Constantine, R. E. Forster, J. Appl. - Physiol. 19, 522 (1964)] with rate constant $k=1.7 \mathrm{sec}^{-1}$ at $p H \quad 8.0$ [D. O Lambeth and G. Palmer, J. Biol. Chem. 248, $6095(1973)]$ and higher at $p H \quad 7.0$ [H. Hartridge and F. J. W. Roughton, Proc. R. Soc. Lond. Ser. A Math. Phys. Sci. 104, 395 (1923)].
15. I. Tyuma, K. Imai, K. Shimizu, Biochemistry 12, 1491 (1973).

16. W. H. Heustis and M. A. Raftery, Biochem. Biophys. Res. Commun. 49, 428 (1972).

17. Q. H. Gibson, J. Biol. Chem. 245, 3285 (1970).

18. J. S. Olson, M. E. Anderson, Q. H. Gibson, ibid. 246, 5919 (1971).

19. Q. H. Gibson, Proc. Natl. Acad. Sci. U.S.A 70, 1 (1973); Y. Henry and R. Cassoly, Biochem. Biophys. Res. Commun. 51, 659 (1973); R. Cassoly, ibid. 55, 1208 (1973).

20. We thank J. M. Salhany, R. D. Gray, and Q. H. Gibson for providing their raw data and for helpful discussions. Supported by the Research Corporation and by NIH grant HL 14322-02 (R. W. Mann, principal investigator)

* Present address: Gordon McKay Laboratories, Harvard University, Cambridge, Mass. 02138 .

$\dagger$ Present address: Biophysical Laboratory, Harvard Medical School, Boston, Mass. 01451.

8 July 1974; revised 10 September 1974

\section{Negative Resistance Characteristic Essential for the \\ Maintenance of Slow Oscillations in Bursting Neurons}

Abstract. Voltage clamping giving step commands reveals a steady-state negative resistance characteristic in the current-voltage curves of Aplysia bursting neurons. This is observed below spike threshold in the unstable range through which the membrane potential slowly oscillates. The negative resistance characteristic underlies this instability and shapes the rapid depolarization-hyperpolarization phase of the cycle. When bursting cells are converted to silent cells (by cooling) the negative resistance is abolished; conversely, when normally silent cells are made to burst (by warming) a negative resistance develops. The presence of negative resistance thus enables the bursting cell to oscillate, whereas its absence precludes such oscillations.

Bursting neurons of the marine mollusk Aplysia californica show a remarkable voltage instability within their range of oscillation. It is impossible, by injecting steady currents, to stabilize the membrane potential of a bursting cell between the extremes of its cycle. The membrane potential will come to a steady value only at the top of the range (with the cell firing steadily), or near the most hyperpolarized point. Studies by Carnevale and Wachtel (1) demonstrated the presence of a steady inward current which depolarizes a bursting cell, and a transient outward current which, triggered by the depolarization, temporarily hyperpolarizes the neuron. However, the simple combination of these reciprocating membrane currents does not explain the voltage instability of these cells. Such a model would be expected to stabilize at any potential within the oscillating range if one injected sufficient hyperpolarizing current to counter the depolarizing influence.

In order to elucidate the mechanisms specifically underlying the oscillations, we took advantage of the fact that the cycle can be reversibly blocked by cooling (2). When the temperature of the ganglion is reduced from $22^{\circ}$ to $10^{\circ} \mathrm{C}$, the intrinsic region of voltage instability is often eliminated, and a stable resting potential is established near the top of the oscillation range. To explore fully the essential features distinguishing warm bursting cells from cells silenced by cooling, we used the voltage clamp technique to examine the current-voltage $(I-V)$ relation of the same cell under both temperature conditions. Intracellular recordings were made from abdominal ganglion bursting cells $\left(\mathrm{L}_{2}\right.$ to $\mathrm{L}_{6}$ ) by using two $\mathrm{KCl}$-filled microelectrodes and standard techniques (3). Our voltage clamp was designed to study small currents ( $0.1 \mathrm{na})$ below spike threshold. Axonal charging currents prohibited the study of events shorter than $100 \mathrm{msec}$ (4), but this response time was sufficient to register the slow currents underlying the oscillation.

We adjusted the voltage clamp to hold the cell at the least negative point in the cycle of oscillation (usually between -25 and $-30 \mathrm{mv}$ ). When the clamp was activated, the holding current achieved a steady inward value 